CERN-TH/96-179

\title{
Topological Defects in Gauge Theories of Open $p$-branes
}

\author{
M. C. Diamantini \\ CERN Theory Division, CH-1211 Geneva 23, Switzerland
}

\begin{abstract}
We study phase transitions induced by topological defects in compact Abelian gauge theories of open $p$-branes in $(\mathrm{d}+1)$ space-time dimensions. Starting from a massive antisymmetric tensor theory for the open $p$-branes we show how the condensation of topological defects can lead to a decoupled phase with a massless tensor coupled to closed $(p-1)$ branes and a massive tensor coupled to open $(p+1)$-branes. We also consider the case, relevant in string theory, in which the boundaries of the $p$-branes are constrained to live on a Dirichlet $n$-brane.
\end{abstract}

CERN - TH/96 - 179

July 1996 
Antisymmetric tensors of rank $(p+1)((p+1)$-forms $)$ have been widely studied in recent years [1], [2], [3], [4], [5]. They arise naturally in constructing Abelian $U(1)$ gauge theories of elementary extended objects (strings, membranes, $\ldots)$ : a $(p+1)$ antisymmetric tensor couples to elementary $p$-branes in the same way as the vector potential one-form in Maxwell theory couples to elementary point-particles (0-branes). Antisymmetric tensors appear in the effective field theories for the low-energy dynamics of strings and in supersymmetric theories [6].

As in the familiar case of QED [7], the compactness of the gauge symmetry implies the existence of topological defects. These play a crucial role in gauge theories and can lead to drastic modifications of the perturbative infrared behaviour of a theory. Both $U(1)$ and $R$ lead to the same perturbative expansion, but in the former case the condensation of topological defects can generate a mass for the gauge fields and cause confinement.

The topological defects are $(\mathrm{d}-p-2)$-dimensional extended objects representing the world-hypervolumes of $(\mathrm{d}-p-3)$-branes (instantons are $(-1)$-branes). The possible phase diagram depends on the space-time dimension and on the dimension of the topological defects. This can be easily seen if we think of compact QED: in 3 dimensions the topological excitations are (-1)-branes (instantons); these are in a plasma phase for all values of the coupling constant, leading to electric charge confinement [7]. In 4 dimensions the topological defects are 0-branes (monopoles) and they condense only above a critical value of the coupling constant.

For closed or infinitely extended $p$-branes the action that describes the model can be written as

$$
\begin{aligned}
S & =\int \frac{(-1)^{p+1}}{g^{2}} d B_{p+1} \wedge * d B_{p+1}+j B_{p+1} \wedge * J_{p+1} \\
& =\int \frac{(-1)^{p+1}}{g^{2}} d B_{p+1} \wedge * d B_{p+1}+j \int_{\text {branes }} B_{p+1},
\end{aligned}
$$

where $B_{p+1}$ is the antisymmetric tensor field, $g$ is a dimensionless coupling constant, $j$ has dimension $\left[m^{\frac{d-2 p-3}{2}}\right]$, and $J_{p+1}$ is a conserved (tensor) current of closed or infinitely extended $p$-branes :

$$
J^{\mu_{1} \ldots \mu_{p+1}}(x)=\int \delta^{d+1}(x-y(\sigma)) d y^{\mu_{1}} \wedge \ldots \wedge d y^{\mu_{p+1}},
$$

where $y(\sigma)$ are the coordinates of the world-volumes of the $p$-branes. The action (11) is the same action as the one that describes the coupling of a Dirichlet $p$-brane to a $(p+1)$ 
form [8]. We will consider the current $J_{p+1}$ as an external probe and ignore the action governing its dynamics.

For $p=1$ and $\mathrm{d}+1=4$ (1) describes the classical interstring action for closed strings [1]. The effect of the presence of topological defects in ([1) has been studied in [4], [9] using a lattice regularization in Euclidean space-time. By means of a duality transformation on the lattice, (1) can be transformed into a theory formulated in terms of integer fields and this formulation can be used to describe the interactions of topological defects. What has been found [4] is that the theory is disordered (condensation of the topological defects) for all couplings if $\mathrm{d}=p+1$, while a critical non-zero coupling exists for $\mathrm{d}=p+2$. When the system is disordered, the effect of the condensation of the topological defects is the confinement of closed $p$-branes.

In this paper we are interested in considering the effects of topological excitations in a $U(1)$ gauge theory for open $p$-branes. The theory we consider is:

$$
\begin{aligned}
S= & \int \frac{(-1)^{p+1}}{g^{2}} d B_{p+1} \wedge * d B_{p+1}+(-1)^{p}\left(m B_{p+1}+\frac{1}{e} d A_{p}\right) \wedge *\left(m B_{p+1}+\frac{1}{e} d A_{p}\right) \\
& +j\left(B_{p+1}+\frac{1}{m e} d A_{p}\right) \wedge * J_{p+1},
\end{aligned}
$$

where $A_{p}$ is an antisymmetric tensor of rank $p, e$ is a dimensionless coupling constant, $m$ is a mass parameter, and $S$ is invariant under the combined gauge symmetry:

$$
\begin{aligned}
B_{p+1} & \rightarrow B_{p+1}+d \Lambda_{p}, \\
A_{p} & \rightarrow A_{p}-m e \Lambda_{p} .
\end{aligned}
$$

The term $\left(B_{p+1}+\frac{1}{m e} d A_{p}\right)$ is itself gauge-invariant; for this reason we do not need the current $J_{p+1}$ to be conserved, and we can couple the theory to open $p$-branes.

Again, for $p=1$ and $\mathrm{d}+1=4$, (3) describes the classical interstring action for open strings [1], [9]. In this case $d A$ is included to ensure gauge invariance at the end-points of the open string. More generally, if we look at (1) we see that the coupling is invariant only if the world-hypervolume of the $p$-brane has no boundary. The antisymmetric tensor $A_{p}$ couples to the boundary and restores gauge invariance.

Also interesting to study is a slight modification of (3), namely: 


$$
\begin{aligned}
S= & \int_{M} \frac{(-1)^{p+1}}{g^{2}} d B_{p+1} \wedge * d B_{p+1}+\int_{\Sigma}(-1)^{p}\left(m B_{p+1}+\frac{1}{e} d A_{p}\right) \wedge *\left(m B_{p+1}+\frac{1}{e} d A_{p}\right) \\
& +\int_{M} j B_{p+1} \wedge * J_{p+1}+\int_{\Sigma} j \frac{1}{m e} A_{p} \wedge * \delta J_{p+1} .
\end{aligned}
$$

Here $M$ indicates the full $(\mathrm{d}+1)$-dimensional space-time, while $\Sigma$ is an infinitely extended $n$-dimensional hypersurface in our space-time $((\mathrm{d}+1) \geq n)$ on which the boundary of the open $p$-brane is constrained to live. In this case $m$ has dimension [mass $\left.\frac{n-d+1}{2}\right]$. We recover (3) when $(\mathrm{d}+1)=n$. The action (5) is a generalization, in flat space-time, of the effective action [10] for Type II superstrings in 10 dimensions in presence of a D-brane (a D-string in the particular case $n=2)$ :

$$
S=\int_{M} d^{10} x \sqrt{g} \frac{1}{2 \lambda^{2}} d B_{2} \wedge * d B_{2}+\int_{\Sigma} d^{2} x \sqrt{g_{\Sigma}} \frac{1}{2 \lambda}\left(B_{2}+d A\right) \wedge *\left(B_{2}+d A\right)
$$

where $\lambda$ is the string coupling constant and $g$ and $g_{\Sigma}$ are the metrics on $M$ and $\Sigma$. D-branes in Type II superstrings are BPS states that carry RR charges [8]. The necessity of objects that carry RR charges comes from string dualities [11]. The end-points of open strings are constrained to live on the brane. This is why the part of the action that contains the $U(1)$ gauge field $A$, which couples to the boundaries of the open string, is defined on the D-brane world-sheet. In our simplified model, the D-brane is considered as a submanifold without dynamics on which the boundaries of the open $p$-branes are constrained to live. This allows us to consider the the generic case of open $p$-branes ending on $n$-branes with the only constraint $(d+1) \geq n \geq p$, without taking into account consistency coming from string theory [8] [12] [13] [14]. Note also that fermion zero-modes in supersymmetric theories may crucially affect the dynamics of topological excitations [15].

We will start with the analysis of the first model (3) and then come back to (5). In (3) the tensor of higher-rank possesses a bare mass term. In fact, up to a gauge transformation, we can rewrite (3) as a generalized version of the Proca Lagrangian for an antisymmetric tensor field $\bar{B}_{p+1}=B_{p+1}+\frac{1}{m e} d A_{p}$ :

$$
\begin{aligned}
S= & \int \frac{(-1)^{p+1}}{g^{2}} d \bar{B}_{p+1} \wedge * d \bar{B}_{p+1}+(-1)^{p} m^{2} \bar{B}_{p+1} \wedge * \bar{B}_{p+1} \\
& +j \bar{B}_{p+1} \wedge * J_{p+1} .
\end{aligned}
$$


In this formulation the higher-rank tensor $B_{p+1}$ has "eaten" the tensor $A_{p}$, which is a generalization of the familiar Higgs-Stückelberg mechanism for vector fields. Correspondingly, (7) describes a generalized "Higgs" phase [9]. When $(\mathrm{d}+1)=4$ and $p=1$ the integral over $B_{2}$ defines the action for the confining string $J_{2}$ [5], [16]. What we want to do now is to take into account the presence of topological defects in the theory with compact symmetry, and study what the effects of their possible condensation are.

In general, when studying the effect of condensation of topological excitations, one starts with a compact theory in the Coulomb phase. The condensation of topological defects, when possible, disorders the system and generates a mass for the gauge field.

We know from the Stückelberg mechanism [17] that the limit in which the mass goes to zero in (17) is singular, because it introduces an additional gauge symmetry, not present in the original theory. The presence of a massless pole in the propagator for the $B_{p+1}$ tensor, in the limit $m \rightarrow 0$, can be seen by adding a gauge-fixing term; in order to recover a gauge-invariant theory in the limiting case, however, it is necessary that the tensor field be coupled to a conserved current, and in our case $J_{p+1}$ is not (current of open $p$-branes). As we will see, there is also in this case the possibility of the appearance of a gauge-invariant massless pole in the propagator of the $B_{p+1}$ tensor. What leads to the zero-mass limit in our theory is the condensation of topological excitations; apparently, in this case, these work in the opposite direction with respect to the general case by cancelling the mass for the gauge field $B_{p+1}$. As we will show, after the condensation of the topological defects, the $B_{p+1}$ tensor couples automatically only to the transverse part of $J_{p+1}$; the longitudinal part decouples and describes a generalized Coulomb gas. The transverse part represents a conserved current and the theory is gauge-invariant.

The topological defects can be treated explicitly by rewriting (3) as:

$$
\begin{aligned}
S= & \int \frac{(-1)^{p+1}}{g^{2}}\left(d B_{p+1}+q t_{p+2}\right) \wedge *\left(d B_{p+1}+q t_{p+2}\right)+ \\
& +(-1)^{p}\left(m B_{p+1}+\frac{1}{e} d A_{p}+\frac{s}{e} t_{p+1}\right) \wedge *\left(m B_{p+1}+\frac{1}{e} d A_{p}+\frac{s}{e} t_{p+1}\right)+ \\
& +j\left(B_{p+1}+\frac{1}{m e} A_{p}\right) \wedge * J_{p+1} .
\end{aligned}
$$

Here $t_{p+2}$ and $t_{p+1}$ are singular forms representing topological defects of charge $q$ and $s$ [3], [5] associated with the two compact gauge fields $B_{p+1}$ and $A_{p}$ : 


$$
* t_{r}=G_{d-r+1}, \quad G_{d-r+1}^{\mu_{1} \ldots \mu_{d-r+1}}=\int \delta^{d+1}(x-\tilde{y}(\tilde{\sigma})) d \tilde{y}^{\mu_{1}} \wedge \ldots \wedge d \tilde{y}^{\mu_{d-r+1}}
$$

Here $\tilde{y}^{\mu}\left(\tilde{\sigma}^{\nu_{1}}, \ldots, \sigma^{\nu_{d-r+1}}\right)$ is an open hypervolume describing the generalization to higherdimensional topological defects of the Dirac string. The boundary of this hypervolume describes the world-hypersurface of the topological defects.

Because the forms $t_{r}$ are singular, their treatment requires a proper regularization. For vector fields we can think of Abelian compact gauge models as the low-energy effective field theory due to spontaneous symmetry breaking of a non-Abelian gauge group [7]. In this case the mass acquired by the broken gauge fields becomes the cut-off for the low energy theory. Another possibility is to use a lattice regularization, which is the route we will eventually choose.

By integrating over the tensor field $A_{p}$ we obtain an effective action for the higher rank-tensor $B_{p+1}$. When the topological defects of $A_{p}$ are dilute, this effective action still possesses a massive pole, while in the case in which these topological defects are in a dense phase, the effective action we get is (ignoring for the moment the coupling with the current of open $p$-branes):

$$
S_{\text {eff }}=\int \frac{(-1)^{p+1}}{g^{2}}\left(d B_{p+1}+q t_{p+2}\right) \wedge *\left(d B_{p+1}+q t_{p+2}\right) .
$$

This is nothing else than the compact version of (11): the mass term for $B_{p+1}$ is no longer present. If we count the degrees of freedom in (7) we obtain $\left(\begin{array}{c}d \\ p+1\end{array}\right)$; this expression can be written as:

$$
\left(\begin{array}{c}
d \\
p+1
\end{array}\right)=\left(\begin{array}{c}
d-1 \\
p+1
\end{array}\right)+\left(\begin{array}{c}
d-1 \\
p
\end{array}\right) .
$$

Equation (11) is the sum of the degrees of freedom of the two massless free theories for the two antisymmetric tensors $B_{p+1}$ and $A_{p}$ : in (7) the tensor $B_{p+1}$ "eats" $A_{p}$ and becomes massive through the Higgs-Stückelberg mechanism. The condensation of the topological defects of $A_{p}$ prevents this mechanism.

In the coupling with the $(p+1)$-current of open $p$-branes, $j \int\left(B_{p+1}+\frac{1}{m e} d A_{p}\right) \wedge * J_{p+1}$, we use the Hodge decomposition for the form $J_{p+1}$ :

$$
J_{p+1}=d \beta_{p}+\delta \alpha_{p+2}
$$


where $\delta$ is the adjoint exterior derivative. The properties of the inner product between forms and the fact that $\delta^{2}=0$ tell us that $d A_{p}$ couples only to $d \beta_{p}:\left(d A_{p}, d \beta_{p}\right)=$ $\left(A_{p}, \delta d \beta_{p}\right)$, where $\delta d \beta_{p}$ is the hypersurface which represents the boundary of the open hypervolume $J_{p+1}$ and (,) denotes the inner product. In the effective action (10), as we will show, the antisymmetric tensor $B_{p+1}$ couples only to the transverse part of $J_{p+1}$, $\delta \alpha_{p+2}$, while the boundaries $\delta d \beta_{p}$ decouple and form a generalized Coulomb gas. This can be represented as a Gaussian integral over a massless $p$-form $A_{p}$ coupling to $\delta d \beta_{p}$.

What happens if also the topological defects of $B_{p+1}$ condense? This can be studied analogously by computing the effective action for $J_{p+1}^{T}$ obtained by integrating over $B_{p+1}$.In the dense phase we obtain a kernel, which can be represented as a Gaussian integral over a new, massive, $(p+2)$-form $C_{p+2}$ coupling to $\alpha_{p+2}$. In this phase we thus obtain two decoupled gauge theories for open $(p-1)$-branes and closed $(p+1)$-branes. These describe $\left(\begin{array}{c}d \\ p+2\end{array}\right)$ massive and $\left(\begin{array}{c}d-1 \\ p\end{array}\right)$ massless degrees of freedom, respectively.

In order to properly derive this mechanism in a specific case of (3), we consider a Euclidean lattice regularization for the case $p=1, \mathrm{~d}+1=4$. In this case $J_{p+1}=J_{2}$ describes the world-sheet of an open string, and its boundary is the closed world-line described by the end-points of the open string.

The continuum non-compact Euclidean action is:

$$
S=\int d^{4} x\left(\frac{1}{2 g^{2}} F_{\mu} F_{\mu}+\frac{1}{4 e^{2}} f_{\mu \nu} f_{\mu \nu}+\frac{\tilde{m}^{2}}{4} B_{\mu \nu} B_{\mu \nu}+\frac{\tilde{m}}{2 e} B_{\mu \nu} f_{\mu \nu}\right),
$$

where $B_{\mu \nu}$ is the antisymmetric Kalb-Ramond tensor, $F_{\mu}=\frac{1}{6} \epsilon_{\mu \nu \alpha \beta} F_{\nu \alpha \beta}$ is the dual of the Kalb-Ramond field strength, $F_{\nu \alpha \beta}=\partial_{[\nu} B_{\alpha \beta]}, f_{\mu \nu}=\partial_{\mu} A_{\nu}-\partial_{\nu} A_{\mu}$ is the Maxwell field strength, and we have renamed the mass parameter as $\tilde{m}$. Equation (12) is invariant under the combined gauge transformation

$$
\begin{aligned}
B_{\mu \nu} & \rightarrow B_{\mu \nu}+\partial_{\mu} \Lambda_{\nu}-\partial_{\nu} \Lambda_{\mu} \\
A_{\mu} & \rightarrow A_{\mu}-\tilde{m} e \Lambda_{\mu} .
\end{aligned}
$$

The lattice we consider is a hypercubic lattice in four Euclidean dimensions, whose sites are denoted by $x$, and lattice spacing $l$. The gauge fields, which are angular variables for the compact theory on the lattice, are associated with the links $(x, \mu)$ between the sites $x$ and $(x+\hat{\mu})$, where $\hat{\mu}$ denotes a unit vector in the direction $\mu$ on the lattice. On the lattice, we define the following forward and backward derivatives and shift operators: 


$$
\begin{array}{ll}
d_{\mu} f(\mathbf{x}) \equiv \frac{f(\mathbf{x}+\hat{\mu} l)-f(\mathbf{x})}{l}, & S_{\mu} f(\mathbf{x}) \equiv f(\mathbf{x}+\hat{\mu} l), \\
\hat{d}_{\mu} f(\mathbf{x}) \equiv \frac{f(\mathbf{x})-f(\mathbf{x}-\hat{\mu} l)}{l}, & \hat{S}_{\mu} f(\mathbf{x}) \equiv f(\mathbf{x}-\hat{\mu} l) .
\end{array}
$$

Summation by parts interchanges the two derivatives, with a minus sign, and the two shift operators. We also introduce the three-index lattice operators [18]:

$$
K_{\mu \nu \alpha}=S_{\mu} \epsilon_{\mu \rho \nu \alpha} d_{\rho}, \quad \hat{K}_{\mu \nu \alpha}=\epsilon_{\mu \nu \rho \alpha} \hat{d}_{\rho} \hat{S}_{\alpha}
$$

These operators are gauge-invariant in the sense that:

$$
\begin{aligned}
& K_{\mu \nu \alpha} d_{\alpha}=K_{\mu \nu \alpha} d_{\nu}=\hat{d}_{\mu} K_{\mu \nu \alpha}=0 \\
& \hat{K}_{\mu \nu \alpha} d_{\alpha}=\hat{d}_{\mu} \hat{K}_{\mu \nu \alpha}=\hat{d}_{\nu} \hat{K}_{\mu \nu \alpha}=0 .
\end{aligned}
$$

Moreover they satisfy the equations:

$$
\begin{aligned}
& \hat{K}_{\mu \nu \alpha} K_{\alpha \lambda \omega}=K_{\mu \nu \alpha} \hat{K}_{\alpha \lambda \omega}=O_{\mu \nu \lambda \omega}= \\
& =-\left(\delta_{\mu \lambda} \delta_{\nu \omega}-\delta_{\mu \omega} \delta_{\nu \lambda}\right) \nabla^{2}+\left(\delta_{\mu \lambda} d_{\nu} \hat{d}_{\omega}-\delta_{\nu \lambda} d_{\mu} \hat{d}_{\omega}\right)+\left(\delta_{\nu \omega} d_{\mu} \hat{d}_{\lambda}-\delta_{\mu \omega} d_{\nu} \hat{d}_{\lambda}\right), \\
& \hat{K}_{\mu \omega \alpha} K_{\omega \alpha \nu}=K_{\mu \omega \alpha} \hat{K}_{\omega \alpha \nu}=2 M_{\mu \nu}=-2\left(\delta_{\mu \nu} \nabla^{2}-d_{\mu} \hat{d}_{\nu}\right) .
\end{aligned}
$$

The expressions $O_{\mu \nu \lambda \omega}$ and $M_{\mu \nu}$ are lattice versions of the Kalb-Ramond and Maxwell kernels, respectively, and $\nabla^{2}=d_{\mu} \hat{d}_{\mu}=\hat{d}_{\mu} d_{\mu}$ is the lattice Laplacian.

The formulation of the theory on the lattice allows a quantitative analysis of the phase diagram and the condensation conditions for the topological excitations. We consider a partition function of the Villain [19] type:

$$
\begin{aligned}
Z(J) & =\sum_{\substack{\left\{\tilde{b}_{\mu\},\left\{\tilde{a}_{\mu \nu\}}\right.}\left\{m_{\mu \nu}\right\} \\
S\right.}} \int_{\frac{-\pi}{l}}^{\frac{\pi}{l}} \mathcal{D} A_{\mu} \mathcal{D} B_{\mu \nu} \exp (-S) \\
S & =\sum_{\mathbf{x}, \mu} \frac{l^{4}}{2 g^{2}}\left(F_{\mu}+\frac{\pi}{l^{2}} \tilde{b}_{\mu}\right)^{2}+\frac{l^{4}}{4} \tilde{m}^{2}\left(B_{\mu \nu}+\frac{2 \pi}{l} m_{\mu \nu}\right)^{2}+\frac{l^{4}}{4 e^{2}}\left(f_{\mu \nu}+\frac{2 \pi}{l^{2}} \tilde{a}_{\mu \nu}\right)^{2} \\
& +\frac{l^{4}}{2 e} \tilde{m}\left(B_{\mu \nu}+\frac{2 \pi}{l} m_{\mu \nu}\right)\left(f_{\mu \nu}+\frac{2 \pi}{l^{2}} \tilde{a}_{\mu \nu}\right)^{2}-i l j\left(B_{\mu \nu}+\frac{1}{\tilde{m} e} f_{\mu \nu}\right) J_{\mu \nu}
\end{aligned}
$$

The dual of $F_{\mu \nu \alpha}$ is expressed in terms of the $K_{\mu \nu \alpha}$ operator as: $F_{\mu}=\frac{1}{2} K_{\mu \nu \alpha} B_{\nu \alpha}$. The integer fields $\tilde{a}_{\mu \nu}, \tilde{b}_{\mu}$ and $m_{\mu \nu}$ ensure the periodicity under the transformations 


$$
A_{\mu} \rightarrow A_{\mu}+\frac{2 \pi}{l} n_{\mu} \quad \text { and } \quad B_{\mu \nu} \rightarrow B_{\mu \nu}+\frac{2 \pi}{l} n_{\mu \nu}
$$

with $n_{\mu}$ and $n_{\mu \nu} \in \mathcal{Z}$. The action in (18) possesses also the gauge symmetry (13) with derivatives substituted by lattice derivatives $d$.

The last term in (18), the coupling with the world-sheet of an open string, plays the role of the (non-local) order parameter, the Wilson "surface" $W_{S}$, for the phase transitions in the theory. $J_{\mu \nu}$ vanishes everywhere but on the plaquettes of the Wilson surface, where it takes the value 1 . The integer $j$ represents the strength of the coupling. The most general case in which the last term in (18) is invariant under (19), is when:

$$
\frac{1}{l \tilde{m} e}=\frac{q}{p} \text { and } j=\tilde{j} p
$$

with $p, q$ and $\tilde{j} \in \mathcal{Z}$ and $(p, q)$ coprime.

Now we decompose the integers $\tilde{a}_{\mu \nu}$ and $\tilde{b}_{\mu}$ as

$$
\begin{aligned}
\tilde{a}_{\mu \nu} & =l\left(d_{\mu} \lambda_{\nu}-d_{\nu} \lambda_{\mu}\right)+a_{\mu \nu}, \\
\tilde{b}_{\mu} & =l K_{\mu \nu \alpha} m_{\nu \alpha}+b_{\mu},
\end{aligned}
$$

and change variables $B_{\mu \nu} \rightarrow B_{\mu \nu}+\frac{2 \pi}{l} m_{\mu \nu}, A_{\mu} \rightarrow A_{\mu}+\frac{2 \pi}{l} \lambda_{\mu}$. The sum over $m_{\mu \nu}$ and $\lambda_{\mu}$ has the effect of shifting the integrals in the partition function from $[-\pi / l, \pi / l]$ to $(-\infty,+\infty)$. We can now perform the Gaussian integrals over the gauge fields to compute the expectation value $\left\langle W_{S}\right\rangle=Z(J) / Z$ of the Wilson surface:

$$
\begin{aligned}
\left\langle W_{S}\right\rangle & =\frac{1}{Z_{\text {top }}} \sum_{\substack{\{c \mu\} \\
\left\{b_{\mu}\right\}}} \exp \left(-S_{\text {top }}-W_{\text {top }}-W_{0}\right) \\
S_{\text {top }} & =\sum_{\mathbf{x}, \mu} \frac{\pi^{2}}{2 g^{2}} b_{\mu} \frac{m^{2} \delta_{\mu \nu}-d_{\mu} \hat{d}_{\nu}}{m^{2}-\nabla^{2}} b_{\nu}+\frac{\pi^{2}}{2 e^{2} l^{2}} c_{\mu} \frac{1}{m^{2}-\nabla^{2}} c_{\mu}-\frac{\pi^{2} m}{l g e} b_{\mu} \frac{1}{m^{2}-\nabla^{2}} c_{\mu}, \\
W_{0} & =\sum_{\mathbf{x}, \mu} \frac{g^{2} j^{2}}{l^{2}} J_{\mu \nu} \frac{1}{m^{2}-\nabla^{2}} J_{\mu \nu}+\frac{2 g^{2} j^{2}}{m^{2} l^{2}} \hat{d}_{\mu} J_{\mu \nu} \frac{1}{m^{2}-\nabla^{2}} \hat{d}_{\rho} J_{\rho \nu}, \\
W_{\text {top }} & =\sum_{\mathbf{x}, \mu} \frac{i \pi j}{l} b_{\mu} \frac{\hat{K}_{\mu \nu \alpha}}{m^{2}-\nabla^{2}} J_{\nu \alpha}+\frac{i \pi j g m}{e l^{2}} c_{\mu} \frac{K_{\mu \nu \alpha}}{m^{2}-\nabla^{2}} J_{\nu \alpha} .
\end{aligned}
$$

Here $c_{\mu}=l K_{\mu \nu \alpha} a_{\nu \alpha}$ is the physical integer degree of freedom that describes the topological defects associated with the integer part of the Maxwell gauge field. It describes closed (or 
infinitely long) strings of magnetic charge: $\hat{d}_{\mu} c_{\mu}=0$. Note that, due to this constraint and to the gauge invariance of $\lambda_{\mu}$ in (21), the set $\left\{c_{\mu}, \lambda_{\mu}\right\}$ describes 6 integer degrees of freedom, which are equivalent to the original antisymmetric integers $\tilde{a}_{\mu \nu}$. The strings $b_{\mu}$ come, instead, from the integer part of $B_{\mu \nu}$; they can be either open or closed. The parameter $m=g \tilde{m}$.

In order to establish the phase structure of the model, we need to analyse the conditions for the condensation of the topological excitations. To this end we shall use the same free-energy arguments as those adopted in the analysis of related $(3+1)$-dimensional models [20] [21], in which the condition for the condensation of strings is established by comparing their self-energy and their entropy.

The entropy of a string of length $L=l N$ can be estimated using the theory of random walks [22] as $\gamma N$ : the parameter $\gamma$ is given roughly by $\gamma=\ln 7$, since at each step the string can choose between 7 different directions. In a dilute instanton approximation, in which all values $c_{\mu}, b_{\mu} \geq 2$ are neglected, it can be proved that the correct value of $\gamma$ is the same for open and closed strings [23]. We will neglect all subdominant functions of $N$, such as a $\ln N$ correction to the entropy. The free energy of a string of length $L=l N$ carrying quantum numbers $c$ and $b$ is thus essentially

$$
F=\left\{\frac{\pi^{2}}{2 g^{2}} G(m l)\left(b-\frac{q}{p} c\right)^{2}-\gamma\right\} N,
$$

where $G(m l)$ is the diagonal element of the lattice kernel $G(\mathbf{x}-\mathbf{y})$ representing the inverse of the operator $\left(1-\frac{\nabla^{2}}{m^{2}}\right)$. Clearly, this diagonal element depends on the dimensionless parameter $(\mathrm{ml})$. Strings condense when the coefficient of $N$ becomes negative. This condensation condition depends crucially upon the integer coprimes $p$ and $q$ : if $p>q$ we will have a phase in which the $c_{\mu}$ form a condensate while the $b_{\mu}$ are in a dilute phase in the regime

$$
\gamma<\frac{\pi^{2} G(m l)}{2 g^{2}}<\frac{p^{2}}{q^{2}} \gamma
$$

If $q>p$ the opposite happens. We will concentrate on the former case, which is by far the most interesting.

When the topological defects $c_{\mu}$ condense, the Wilson surface expectation value takes the form: 


$$
\begin{aligned}
\left\langle W_{S}\right\rangle & =\frac{1}{Z_{\mathrm{top}}} \sum_{\left\{b_{\mu}\right\}} \exp \left(-S_{\mathrm{top}}-W_{\mathrm{top}}-W_{0}\right) \\
S_{\mathrm{top}} & =\sum_{\mathbf{x}, \mu}-\frac{\pi^{2}}{2 g^{2}} Q \frac{1}{\nabla^{2}} Q, \\
W_{0} & =\sum_{\mathbf{x}, \mu}-\frac{g^{2} j^{2}}{l^{2}} J_{\mu \nu}^{\mathrm{T}} \frac{1}{\nabla^{2}} J_{\mu \nu}^{\mathrm{T}}-\frac{2 g^{2} j^{2}}{m^{2} l^{2}} \hat{d}_{\mu} J_{\mu \nu} \frac{1}{\nabla^{2}} \hat{d}_{\rho} J_{\rho \nu}, \\
W_{\mathrm{top}} & =\sum_{\mathbf{x}, \mu}-\frac{i \pi j}{l} b_{\mu} \frac{\hat{K}_{\mu \nu \alpha}}{\nabla^{2}} J_{\nu \alpha}^{\mathrm{T}} .
\end{aligned}
$$

Here $Q=l \hat{d}_{\mu} b_{\mu}$ represent the monopoles that live at the end-points of the $b_{\mu}$ strings and $J_{\mu \nu}^{\mathrm{T}}=-\frac{1}{2} \frac{O_{\mu \nu \alpha \beta}}{\nabla^{2}} J_{\alpha \beta}$ is the transverse projection of the $J_{\mu \nu}$ current. In (24) the longitudinal degrees of freedom of $J_{\mu \nu}$ form a generalized Coulomb gas and are completely decoupled from the transverse degrees of freedom. The transverse contribution can be obtained starting from a compact Kalb-Ramond action

$$
S=\sum_{\mathbf{x}, \mu} \frac{l^{4}}{2 g^{2}}\left(F_{\mu}+\frac{\pi}{l^{2}} \tilde{b}_{\mu}\right)^{2}-i l j B_{\mu \nu} J_{\mu \nu}^{\mathrm{T}},
$$

while the Coulomb gas for the boundaries can be obtained from an action (11) with $p=0$ (non compact QED) coupled to $\hat{d}_{\mu} J_{\mu \nu}$. These two gauge theories for $J_{\mu \nu}^{\mathrm{T}}$ and $\hat{d}_{\mu} J_{\mu \nu}$ are completely independent.

The monopoles $Q$ are always in a plasma phase [7], [1] (which is not in contrast with saying that the $c_{\mu}$ strings condense first, because it is their condensation that frees the monopoles at the end-points of the $b_{\mu}$ strings). In order to evaluate the effects of the condensation of monopoles, we choose the external probe as $J_{\mu \nu}=l d_{\mu} J_{\nu}+l K_{\mu \nu \alpha} \phi_{\alpha}$, with $J_{\mu}$ and $\phi_{\mu}$ integer, so that $J_{\mu \nu}^{\mathrm{T}}=l K_{\mu \nu \alpha} \phi_{\alpha}$. Repeating Polyakov's calculation [7], we see that monopole condensation changes the transverse contribution to

$$
W_{0}=\sum_{\mathbf{x}, \mu}-2 g^{2} j^{2} \phi_{\mu} \phi_{\mu}+\ldots ;
$$

$\phi_{\mu}$ is the dual of the volume form $\alpha_{3}$ that appears in the Hodge decomposition of the current $J_{\mu \nu}$ so that (25) describes the volume law in the case of closed strings. This result is correct in the limit in which the mass $\bar{m}$ generated by the monopole condensation [7] is very large. Away from this limit, we would have had:

$$
W_{0}=\sum_{\mathbf{x}, \mu} \frac{-g^{2} j^{2}}{l^{2}} J_{\mu \nu}^{T} \frac{1}{\bar{m}^{2}-\nabla^{2}} J_{\mu \nu}^{T}-2 g^{2} j^{2} \phi_{\mu} \frac{\bar{m}^{2}}{\bar{m}^{2}-\nabla^{2}} \phi_{\mu}+\ldots
$$


This term can be obtained starting from an action of type (7), with $p=2$ and the identification $J_{3}=* \phi_{1}, \delta J_{3}=J_{2}^{T}$. The appearance of a massive 3 -index tensor is an example of the more general Julia-Toulouse mechanism [5] and can be interpreted as string confinement. After condensation of topological defects, our original gauge model for the interactions of open 1-branes can be rewritten as the sum of two completely decoupled theories, one formulated in terms of a massless vector that describes the interactions of 0-branes (particles) and the other formulated in terms of a massive 3-index tensor that describes the interactions of open 2-branes (membranes).

This example can be easily generalized to the case of $(d+1)$ dimensions and to generic $p$. The topological defects will be, in general, objects with higher dimensions with respect to the case in the example. For this reason, the condensation conditions will be much more complicated. In the following we assume that there is a phase in which the topological defects of the lower-rank tensor condense, and show that, in this case, the result is analogous to the above example.

To this end we start from a generalized version of (18) (we consider $l=1$ in order to simplify the notation):

$$
\begin{aligned}
& Z(J)=\sum_{\substack{\left\{\tilde{b}_{\left.\mu_{1} \ldots \mu_{d-p-1}\right\},\left\{\tilde{a} \mu_{1} \ldots \mu_{p+1}\right\}}\right\} \\
\left\{m_{\left.\mu_{1} \ldots \mu_{p+1}\right\}}\right.}} \int_{-\pi}^{\pi} \mathcal{D} A_{\mu_{1} \ldots \mu_{p}} \mathcal{D} B_{\mu_{1} \ldots \mu_{p+1}} \exp (-S) \\
& S=\sum_{\mathbf{x}, \mu} \frac{1}{4(d-1) ! g^{2}}\left(F_{\mu_{1} \ldots \mu_{d-p-1}}+2 \pi \tilde{b}_{\mu_{1} \ldots \mu_{d-p-1}}\right)^{2}+ \\
& +\frac{1}{4} \tilde{m}^{2}\left(B_{\mu_{1} \ldots \mu_{p+1}}+2 \pi m_{\mu_{1} \ldots \mu_{p+1}}\right)^{2}+\frac{1}{4 e^{2}}\left(f_{\mu_{1} \ldots \mu_{p+1}}+2 \pi \tilde{a}_{\mu_{1} \ldots \mu_{p+1}}\right)^{2}+ \\
& +\frac{1}{2 e} \tilde{m}\left(B_{\mu_{1} \ldots \mu_{p+1}}+2 \pi m_{\mu_{1} \ldots \mu_{p+1}}\right)\left(f_{\mu_{1} \ldots \mu_{p+1}}+2 \pi \tilde{a}_{\mu_{1} \ldots \mu_{p+1}}\right)^{2}+ \\
& -i j\left(B_{\mu_{1} \ldots \mu_{p+1}}+\frac{1}{\tilde{m} e} f_{\mu_{1} \ldots \mu_{p+1}}\right) J_{\mu_{1} \ldots \mu_{p+1}}
\end{aligned}
$$

where $e$ and $g$ are still dimensionless coupling constants. $F_{\mu_{1} \ldots \mu_{d-p-1}}=K_{\mu_{1} \ldots \mu_{d}} B_{\mu_{d-p} \ldots} B_{\mu_{d}}$ is the generalization of the dual of the higher-rank tensor field strength, with $K_{\mu_{1} \ldots \mu_{d}}=$ $S_{\mu_{1}} \epsilon_{\mu_{1} \nu \mu_{2} \ldots \mu_{d}} d_{\nu}\left(\hat{K}_{\mu_{1} \ldots \mu_{d}}=\epsilon_{\mu_{1} \ldots \nu \mu_{d}} \hat{d}_{\nu} \hat{S}_{\mu_{d}}\right)$. The $K$ operators satisfy the equation:

$$
\begin{aligned}
& \hat{K}_{\mu_{1} \ldots \mu_{r} \alpha_{r+1} \ldots \alpha_{d}} K_{\alpha_{r+1} \ldots \alpha_{d} \nu_{1} \ldots \nu_{r}}=K_{\mu_{1} \ldots \mu_{r} \alpha_{r+1} \ldots \alpha_{d}} \hat{K}_{\alpha_{r+1} \ldots \alpha_{d} \nu_{1} \ldots \nu_{r}}=O_{\mu_{1} \ldots \mu_{r} \nu_{1} \ldots \nu_{r}}= \\
& =(d-r-2) !(r+1) !\left[-\delta_{\mu_{1} \nu_{1}} \ldots \delta_{\mu_{r} \nu_{r}} \nabla^{2}+(r+1) d_{\mu_{1}} \hat{d}_{\nu_{1}} \delta_{\mu_{2} \nu_{2}} \ldots \delta_{\mu_{r} \nu_{r}}\right] .
\end{aligned}
$$


With a generalization of (21) we extend the integrals from $[-\pi, \pi]$ to $(-\infty,+\infty)$ and, performing the integral over $A_{\mu_{1} \ldots \mu_{p}}$, we obtain:

$$
\begin{aligned}
& Z(J)=\sum_{\left\{b_{\mu_{1} \ldots \mu_{d-p-1}}\right\},\left\{c_{\mu_{1} \ldots \mu_{d-p-1}}\right\}} \int_{-\infty}^{\infty} \mathcal{D} B_{\mu_{1} \ldots \mu_{p+1}} \exp (-S) \\
& S=\sum_{\mathbf{x}, \mu} \frac{1}{4(d-1) ! g^{2}}\left(F_{\mu_{1} \ldots \mu_{d-p-1}}+2 \pi b_{\mu_{1} \ldots \mu_{d-p-1}}\right)^{2}-i j B_{\mu_{1} \ldots \mu_{p+1}} J_{\mu_{1} \ldots \mu_{p+1}} \\
& -\frac{m^{2}}{4 g^{2}} B_{\mu_{1} \ldots \mu_{p+1}} \frac{O_{\mu_{1} \ldots \mu_{p+1} \nu_{1} \ldots \nu_{p+1}}}{\nabla^{2}} B_{\nu_{1} \ldots \nu_{p+1}}-\frac{\pi^{2}}{e^{2}} c_{\mu_{1} \ldots \mu_{d-p-1}} \frac{1}{\nabla^{2}} c_{\nu_{1} \ldots \nu_{d-p-1}}+ \\
& -\frac{m}{g e} B_{\mu_{1} \ldots \mu_{p+1}} \frac{K_{\mu_{1} \ldots \mu_{p+1} \nu_{p+2} \ldots \nu_{d}}}{\nabla^{2}} c_{\nu_{p+2} \ldots \nu_{d}}-i j \hat{d}_{\alpha} B_{\alpha \mu_{2} \ldots \mu_{p+1}} \frac{1}{\nabla^{2}} \hat{d}_{\beta} J_{\beta \mu_{2} \ldots \mu_{p+1}}+ \\
& +\frac{j^{2}(p+1) g^{2}}{m^{2} e^{2}} \hat{d}_{\alpha} J_{\alpha \mu_{2} \ldots \mu_{p+1}} \frac{1}{\nabla^{2}} \hat{d}_{\beta} J_{\beta \mu_{2} \ldots \mu_{p+1}} .
\end{aligned}
$$

The terms that describe the coupling of $B_{p+1}$ to $J_{p+1}$ can be written as

$$
\begin{aligned}
& +i j B_{\mu_{1} \ldots \mu_{p+1}} J_{\mu_{1} \ldots \mu_{p+1}}+i j \hat{d}_{\alpha} B_{\alpha \mu_{2} \ldots \mu_{p+1}} \frac{1}{\nabla^{2}} \hat{d}_{\beta} J_{\beta \mu_{2} \ldots \mu_{p+1}}= \\
& =-\frac{i j}{(d-p-1) !(p+1)} B_{\mu_{1} \ldots \mu_{p+1}} \frac{O_{\mu_{1} \ldots \mu_{p+1} \nu_{1} \ldots \nu_{p+1}}}{\nabla^{2}} J_{\nu_{1} \ldots \nu_{p+1}}=i j B_{\mu_{1} \ldots \mu_{p+1}} J_{\mu_{1} \ldots \mu_{p+1}}^{T} .
\end{aligned}
$$

$-\frac{1}{(d-p-1) !(p+1)} \frac{O_{\mu_{1} \ldots \mu_{p+1} \nu_{1} \ldots \nu_{p+1}}}{\nabla^{2}}$ is the transverse projection operator. As we can see from (29), the higher-rank tensor couples only to the transverse part of the current $J_{p+1}$ and not to its boundary.

The condensation of the topological excitations $c_{\nu_{1} \ldots \nu_{d-p-1}}$ leads to a term

$$
\frac{m^{2}}{4 g^{2}} B_{\mu_{1} \ldots \mu_{p+1}} \frac{O_{\mu_{1} \ldots \mu_{p+1} \nu_{1} \ldots \nu_{p+1}}}{\nabla^{2}} B_{\nu_{1} \ldots \nu_{p+1}}
$$

which cancels the corresponding one in (28). We are thus left with:

$$
\begin{aligned}
& Z(J)=\sum_{\left\{b_{\left.\mu_{1} \ldots \mu_{d-p-1}\right\}}\right.} \int_{-\infty}^{\infty} \mathcal{D} B_{\mu_{1} \ldots \mu_{p+1}} \exp (-S) \\
& S=\sum_{\mathbf{x}, \mu} \frac{1}{4(d-1) ! g^{2}}\left(F_{\mu_{1} \ldots \mu_{d-p-1}}+2 \pi b_{\mu_{1} \ldots \mu_{d-p-1}}\right)^{2}+ \\
& -i j B_{\mu_{1} \ldots \mu_{p+1}} J_{\mu_{1} \ldots \mu_{p+1}}^{T}+\frac{j^{2}(p+1) g^{2}}{m^{2} e^{2}} \hat{d}_{\alpha} J_{\alpha \mu_{2} \ldots \mu_{p+1}} \frac{1}{\nabla^{2}} \hat{d}_{\beta} J_{\beta \mu_{2} \ldots \mu_{p+1}} .
\end{aligned}
$$


The action (30) describes the theory for a compact antisymmetric tensor of rank $(p+1)$ coupled to a conserved current $J_{\mu_{1} \ldots \mu_{p+1}}^{T}$. The last term is a generalized Coulomb gas for the boundaries of $J_{\mu_{1} \ldots \mu_{p+1}}$. Except for the last term, (30) is exactly the theory studied in [4]. When the system is disordered, with the same steps as in the example, we can rewrite the theory as the sum of two decoupled non-compact theories: one massless for closed $(p-1)$-branes and one massive for open $(p+1)$-branes.

The generalization to the model (5) is easy at this point. The lattice partition function is

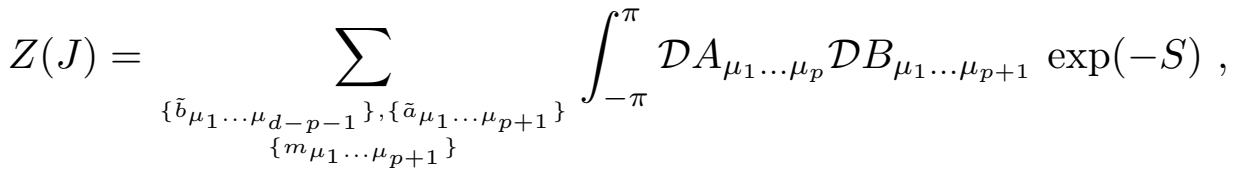

$$
\begin{aligned}
& S=\sum_{\mathbf{x}, \mu}^{M} \frac{1}{4(d-1) ! g^{2}}\left(F_{\mu_{1} \ldots \mu_{d-p-1}}+2 \pi \tilde{b}_{\mu_{1} \ldots \mu_{d-p-1}}\right)^{2}-i j B_{\mu_{1} \ldots \mu_{p+1}} J_{\mu_{1} \ldots \mu_{p+1}}+ \\
& +\sum_{\mathbf{x}, \mu}^{\Sigma} \frac{1}{4} \tilde{m}^{2}\left(B_{\mu_{1} \ldots \mu_{p+1}}+2 \pi m_{\mu_{1} \ldots \mu_{p+1}}\right)^{2}+\frac{1}{4 e^{2}}\left(f_{\mu_{1} \ldots \mu_{p+1}}+2 \pi \tilde{a}_{\mu_{1} \ldots \mu_{p+1}}\right)^{2}+ \\
& +\frac{1}{2 e} \tilde{m}\left(B_{\mu_{1} \ldots \mu_{p+1}}+2 \pi m_{\mu_{1} \ldots \mu_{p+1}}\right)\left(f_{\mu_{1} \ldots \mu_{p+1}}+2 \pi \tilde{a}_{\mu_{1} \ldots \mu_{p+1}}\right)^{2}+ \\
& +i j \frac{(p+1)}{\tilde{m} e} A_{\mu_{1} \ldots \mu_{p}} \hat{d}_{\mu} J_{\mu \mu_{1} \ldots \mu_{p}}
\end{aligned}
$$

where $M$ indicates that the first sum is extended over the entire space-time, while $\Sigma$ indicates that the sum is defined on an $n$-brane. As we said before, we must have $(d+1) \geq$ $n \geq p$.

The topological defects associated with the lower-rank tensor live now on the $n$-brane. With the same analysis as before, when they condense we obtain:

$$
\begin{aligned}
& Z(J)=\sum_{\left\{b_{\left.\mu_{1} \ldots \mu_{d-p-1}\right\}}\right.} \int_{-\infty}^{\infty} \mathcal{D} B_{\mu_{1} \ldots \mu_{p+1}} \exp (-S), \\
& S=\sum_{\mathbf{x}, \mu}^{M} \frac{1}{4(d-1) ! g^{2}}\left(F_{\mu_{1} \ldots \mu_{d-p-1}}+2 \pi b_{\mu_{1} \ldots \mu_{d-p-1}}\right)^{2}-i j B_{\mu_{1} \ldots \mu_{p+1}} J_{\mu_{1} \ldots \mu_{p+1}}^{T}+ \\
& +\sum_{\mathbf{x}, \mu}^{\Sigma} \frac{j^{2}(p+1) g^{2}}{m^{2} e^{2}} \hat{d}_{\alpha} J_{\alpha \mu_{2} \ldots \mu_{p+1}} \frac{1}{\nabla^{2}} \hat{d}_{\beta} J_{\beta \mu_{2} \ldots \mu_{p+1}} .
\end{aligned}
$$


Again, these are the same result as in (30), the only difference being that now the theory for the boundaries is constrained to live on the $n$-brane. When the topological defects in (32) condense we will have a theory for open $(p+1)$-branes defined in all space-time $M$, and a theory for closed $(p-1)$-branes on the $n$-brane.

\section{Acknowledgements}

We acknowledge helpful discussions with A. Giveon, S.J. Rey and C.A. Trugenberger. 


\section{References}

[1] V.I. Ogievetsky and I.V. Polubarinov, Sov. J. Nucl. Phys. 4 (1967) 156; M. Kalb and P. Ramond, Phys. Rev. D9 (1974) 2273.

[2] Y. Nambu, Phys. Rep. 23 (1976) 250; E. Cremmer and J. Scherk, Nucl. Phys. B72 (1974) 117; D.Z. Freedman and P.K. Townsend, Nucl. Phys. B177 (1981) 282.

[3] R.I. Nepomechie, Phys. Rev. D31 (1984) 1921; C. Teitelboim, Phys. Lett. B167 (1986) 63; C. Teitelboim, Phys. Lett. B167 (1986) 69.

[4] R. Savit, Phys. Rev. Lett. 39 (1977) 55; P. Orland, Nucl. Phys. B205[FS5] (1982) 107.For a review see: R. Savit, Rev. Mod. Phys. 52 (1980) 453.

[5] F. Quevedo and C.A. Trugenberger, CERN-TH/96-109, hep-th/9604196.

[6] M. Green, J. Schwarz and E. Witten, "Superstring Theory", Cambridge University Press, Cambridge (1987).

[7] For a review see: A.M. Polyakov, "Gauge Fields and Strings", Harwood Academic Publishers, Chur (1987).

[8] J. Polchinski, S. Chaudhuri and C.V. Johnson, "Notes on D-branes", hep-th/9602052 and references therein.

[9] S.J. Rey, Phys. Rev. D10 (1989) 3396.

[10] E. Witten, IASSNS-HEP-95-83, hep-th/9510135.

[11] C.M. Hull and P.K. Townsend, Nucl. Phys. B438 (1995) 109; E. Witten, Nucl. Phys. B438 (1995) 85; A. Strominger, hep-th/9504090.

[12] M.Douglas, preprint RU-95-92, hep-th/9512077.

[13] A. Strominger, preprint hep-th/9512059.

[14] P.K. Townsend, preprint DAMTP-R-95-59, hep-th/9512062.

[15] We thank S.J. Rey for pointing this out.

[16] A.M. Polyakov, hep-th/9607049.

[17] For a review see: C. Itzykson and J.B. Zuber, "Quantum Field Theory", McGraw-Hill, New York (1980).

[18] M.C. Diamantini, P. Sodano and C.A. Trugenberger, preprint CERN-TH/95-294, to appear in Nucl. Phys. B.

[19] For a review see: H. Kleinert, "Gauge Fields in Condensed Matter", World Scientific, Singapore (1989).

[20] S. Elitzur, R. Pearson and J. Shigemitsu, Phys. Rev. D19 (1979) 3698; D. Horn, $\quad$ M. Weinstein and S. Yankielowicz, Phys. Rev D19 (1979) 3715; A. Guth, A. Ukawa and P. Windey, Phys. Rev. D21 (1980) 1013.

[21] J.L. Cardy and E. Rabinovici, Nucl. Phys. B205 [FS5] (1982) 1; J.L. Cardy, Nucl. Phys. B205 [FS5] (1982) 17; A. Shapere and F. Wilczek, Nucl. Phys. B320 (1989) 669.

[22] For a review see: C. Itzykson and J.M. Drouffe, "Statistical Field Theory", Cambridge University Press, Cambridge (1989).

[23] M.B. Einhorn and R. Savit, Phys. Rev. D19 (1979) 1198. 